\title{
Chapter 25. Drug therapy in CKD
}

- Drugs mainly eliminated by the kidney may increase blood concentrations and exert adverse effects more frequently when they are used in cases of reduced kidney function.

- Dose reduction or prolongation of the interval between administration is necessary in proportion to declining kidney function.

- Use of some antimicrobial agents and NSAIDs may injure the kidney in CKD patients, particularly in the elderly.

- Acute kidney injury due to contrast media occurs more frequently in CKD, diabetic, or elderly patients.

- Allopurinol is reduced in dosage or discontinued in cases of reduced kidney function.

\section{Drug therapy in CKD}

- In reduced kidney function, drugs eliminated by the kidney are not fully metabolized and excreted, resulting in drug accumulation in the blood, which increases the risk of adverse effects.

- In the case of reduced kidney function, the dose or interval of administration of the drug is adjusted according to the eGFR level.
Nonsteroid anti-inflammatory drugs (NSAIDs)

- Administration of NSAIDs may further deteriorate kidney function.

- There are risk factors that facilitate side effects of NSAIDs on the kidney (Table 25-1).

- NSAIDs may cause acute renal failure, water and Na retention, hypertension, hyponatremia, hyperkalemia, interstitial nephritis, or nephrotic syndrome.

- COX-2 inhibitors may also injure the kidney, like conventional NSAIDs.

- NSAIDs should be discontinued immediately when drug-induced acute kidney injury is observed.

\section{Antimicrobial agents}

- Most antimicrobial agents are eliminated by the kidney, so they are reduced in dosage in cases of reduced GFR.

- If the therapeutic concentration of the drug in serum is close to the toxic range, therapeutic drug monitoring (TDM) is desirable. 
Table 25-1 Risk factors for NSAID-induced kidney damage

\begin{tabular}{ll}
\hline Low renal blood flow & Low plasma volume \\
\hline Elderly & Congestive heart failure \\
Hypertension & Nephrotic syndrome \\
CKD & Liver cirrhosis \\
Dehydration & Low ECFV \\
DM & Diuretics \\
\hline
\end{tabular}

Representative drugs that require TDM

(1) Aminoglycoside: acute tubular necrosis occurs with an incidence of $10-20 \%$.

(2) Vancomycin: interstitial nephritis may occur. It is generally desirable that the trough level is maintained at $10 \mu \mathrm{g} / \mathrm{mL}$ or less. Its dosage is determined in accordance with renal function and severity of infection.

Antimycotic agents and antivirus agents that require caution

(1) Amphotericin B: nephrotoxic.

(2) Antivirus agents (acyclovir, ganciclovir, etc.): psychosis and kidney injury may occur.

\section{Antihyperuricemia agents}

- Hyperuricemia is a risk factor for kidney dysfunction and atherosclerosis. Hyperuricemia is preferably treated even without gouty attacks. The target for the serum uric acid level is less than $9.0 \mathrm{mg} / \mathrm{dL}$, but reducing the serum uric acid level to quickly may induce a gouty attack.

- Allopurinol: An inhibitor of uric acid synthesis. In the case of reduced kidney function, allopurinol may cause adverse reactions more frequently and may cause prolonged hypouricemia. Start with a low dosage, if administered. The incidence of side effects is high (4\%), and severe adverse reactions such as hypersensitivity reaction (including Stevens-Johnson syndrome), agranulocytosis, and hypersensitivity vasculitis may occur. A dosage of less than $50 \mathrm{mg}$ /day is safely administered when the GFR is less than $30 \mathrm{~mL} / \mathrm{min} / 1.73 \mathrm{~m}^{2}$.

- Benzbromarone: This agent promotes urinary excretion of uric acid by inhibiting reabsorption of uric acid at proximal tubules. It works less well in reduced GFR and may aggravate kidney function unless sufficient diuresis ( $2 \mathrm{~L} /$ day or more) and alkalization of urine are implemented. This agent increases uric acid excretion in the urine and may promote uric acid stone formation. It may be used in combination with Uralyt or sodium bicarbonate to keep the $\mathrm{pH}$ of urine between 6.2 and 6.8. Uralyt contains potassium, which may cause hyperkalemia. Contraindications for benzbromarone are urinary stones, severe renal insufficiency, or liver dysfunction.

\section{$\mathrm{H}_{2}$ blockers}

- $\mathrm{H}_{2}$ blockers that are used for the treatment of gastric ulcer or chronic gastritis are eliminated by the kidney. The blood concentrations are elevated in kidney dysfunction and may bring about adverse effects such as granulocytopenia or pancytopenia in CKD patients.

- Lafutidine, an $\mathrm{H}_{2}$ blocker, is metabolized primarily in the liver and most of the agent is excreted in bile. Thus, the dose of lafutidine should not be reduced, even in the case of reduced kidney function.

\section{Anticancer drugs}

- Dose adjustment of anticancer drugs is made according to body surface area, but in cases of reduced kidney function it may be necessary to further consider the dosage. In some cases, it is necessary to adjust the dosage according to GFR.

- Cisplatin and other anticancer drugs are highly likely to injure the kidney, thus requiring careful monitoring of kidney function.

- The dose of carboplatin is generally determined based on GFR using Calvert's formula. Replacement of GFR with Ccr would provide excess dosage, potentially causing severe side effects. It is therefore important to adjust the dosage by using an estimated GFR equation.

\section{Calvert's formula \\ Dose of carboplatin $(\mathrm{mg} / \mathrm{body})=$ area under the curve $($ AUC) $(\mathrm{mg} / \mathrm{mL} \times \mathbf{m i n}) \times($ GFR +25$)$}

\section{Contrast media}

- Contrast medium-induced nephropathy is defined as the following: serum creatinine level is increased by $25 \%$ or more, or by $0.5 \mathrm{mg} / \mathrm{dL}$ or more, within $48-72 \mathrm{~h}$ after contrast medium administration. 
Table 25-2 High-risk factors for contrast-media nephropathy

\begin{tabular}{l}
\hline Low GFR \\
Dehydration \\
DM \\
Elderly \\
Congestive heart failure \\
Multiple myeloma \\
Peritoneal dialysis \\
Drugs \\
Diuretics (furosemide, mannitol) \\
NSAIDs \\
Biguanide \\
Antihypertensives \\
Dipyridamole \\
Bosentan \\
Aminoglycosides \\
Vancomycin \\
Amphotericin B
\end{tabular}

- The incidence of contrast media-induced nephropathy is reported to be $1-6 \%$.

- Contrast media-induced nephropathy was reported to occur in $40 \%$ of the individuals in a high-risk group (risk factors are shown in Table 25-2). Therefore, in a high-risk group, examinations employing a contrast medium are done only when the advantages of image testing outweigh the disadvantages or risks of contrast-
Table 25-3 Preventive strategy for contrast-induced nephropathy without hemodialysis

- Twelve hours prior to and after the contrast examination, infuse saline at $1 \mathrm{~mL} / \mathrm{kg}$ per h. (Better with $0.9 \%$ than $0.45 \% \mathrm{NaCl}$.) Drink water if not infused

- Preventive use of N-acetlylcysteine

- Avoid or stop diuretics and NSAIDs

- Strict monitoring of renal function in high-risk cases

- Avoid frequent use (interval should be at least 1-2 weeks)

- There is no evidence supporting the preventive effect of removal of contrast by hemodialysis. Hemodialysis may instead induce hypotension, and further decrease kidney function

- Hemofiltration or hemodialysis (hemodiafiltration) may improve long-term kidney function or life expectancy through the improvement of general circulatory condition

medium nephropathy. The availability of alternative imaging should be considered. In addition, FDA indicates that MRI using gadolinium as a contrast medium in CKD patients may be related to the development of nephrogenic systemic fibrosis/nephrogenic fibrosing dermopathy (NSF/NFD). Careful attention is required in the application of gadoliniumenhanced MRI.

- Several prophylactic measures against contrast-media nephropathy have been propounded (Table 25-3). 